\title{
Temporal changes in innate immune signals in a rat model of alcohol withdrawal in emotional and cardiorespiratory homeostatic nuclei
}

Kate Freeman ${ }^{1}$, Anthony Brureau', Rajanikanth Vadigepalli ${ }^{1}$, Mary M Staehle ${ }^{2}$, Melanie M Brureau', Gregory E Gonye ${ }^{1}$, Jan B Hoek ${ }^{1}$ D Craig Hooper ${ }^{3}$ and James S Schwaber ${ }^{\text {* }^{*}}$

\begin{abstract}
Background: Chronic alcohol use changes the brain's inflammatory state. However, there is little work examining the progression of the cytokine response during alcohol withdrawal, a period of profound autonomic and emotional upset. This study examines the inflammatory response in the central nucleus of the amygdala (CeA) and dorsal vagal complex (DVC), brain regions neuroanatomically associated with affective and cardiorespiratory regulation in an in vivo rat model of withdrawal following a single chronic exposure.

Methods: For qRT-PCR studies, we measured the expression of TNF- $a$, NOS-2, CCI2 (MCP-1), MHC II invariant chain CD74, and the TNF receptor Tnfrsfia in CeA and DVC samples from adult male rats exposed to a liquid alcohol diet for thirty-five days and in similarly treated animals at four hours and forty-eight hours following alcohol withdrawal. ANOVA was used to identify statistically significant treatment effects. Immunohistochemistry (IHC) and confocal microscopy were performed in a second set of animals during chronic alcohol exposure and subsequent 48-hour withdrawal.

Results: Following a chronic alcohol exposure, withdrawal resulted in a statistically significant increase in the expression of mRNAs specific for innate immune markers C CI2, TNF-a, NOS-2, Tnfrsf1a, and CD74. This response was present in both the CeA and DVC and most prominent at 48 hours. Confocal $\mathrm{HC}$ of samples taken 48 hours into withdrawal demonstrate the presence of TNF-a staining surrounding cells expressing the neural marker NeuN and endothelial cells colabeled with ICAM-1 (CD54) and RECA-1, markers associated with an inflammatory response. Again, findings were consistent in both brain regions.

Conclusions: This study demonstrates the rapid induction of CCI2, TNF-a, NOS-2, Tnfrsfla and CD74 expression during alcohol withdrawal in both the CeA and DVC. IHC dual labeling showed an increase in TNF-a surrounding neurons and ICAM-1 on vascular endothelial cells 48 hours into withdrawal, confirming the inflammatory response at the protein level. These findings suggest that an abrupt cessation of alcohol intake leads to an acute central nervous system (CNS) inflammatory response in these regions that regulate autonomic and emotional state.
\end{abstract}

Keywords: Alcohol, Withdrawal, Inflammation, TNF-alpha, MCP-1, Gene expression, IHC

\footnotetext{
* Correspondence: James.Schwaber@jefferson.edu

'Daniel Baugh Institute for Functional Genomics and Computational Biology, Department of Pathology, Anatomy and Cell Biology, Thomas Jefferson University, 1020 Locust Street, Philadelphia, PA 19107, USA

Full list of author information is available at the end of the article
} 


\section{Background}

The relationship between alcohol use, withdrawal and brain inflammation is complex and of considerable interest in light of the high incidence of alcohol use and misuse and the potential impact of alcohol-mediated brain inflammation on neurologic and emotional health [1]. Recent evidence suggests that chronic alcohol exposure alters neuroimmune function, creating a proinflammatory state [2]. For example, astrocytes isolated from the cerebral cortex of alcohol-exposed rats show increased inductible nitric oxide synthase (NOS-2), cyclooxygenase-2, (COX-2) and IL-1 $\beta$ levels in culture [3]. Moreover, 10-day intragastic alcohol exposure amplifies and prolongs the brain's production of TNF- $\alpha$, monocyte chemotactic protein-1 (MCP-1) and IL-1 $\beta$ in response to a peripheral lipopolysaccharide (LPS) injection in rats [4]. The possibility that alcohol could have direct effects on central nervous system (CNS)-resident cells is raised by the finding that alcohol causes a doseand time-dependent induction of TNF- $\alpha$ and Il- $1 \beta$ in cultured microglia [5]. Studies of postmortem brain samples suggest that these findings are relevant to human neuropathology. Histologic changes in microglia, increases in MCP-1 mRNA levels [6], and altered transcriptional regulation of NF-kB [7] have been identified in brain tissue from alcoholics.

Together, these studies suggest that alcohol exposure affects innate immune system function. Normally, the innate immune system protects vertebrates from infection by reacting to nonspecific signals of infection, cellular stress and injury. If such signals, like abnormal lipids, reactive oxygen or nitrogen species, nucleic acids or other cellular debris are encountered, cells of the innate immune system activate various toll-like receptors, downstream JAK-Stat and MAPK signaling pathways [8], biosynthetic processes [9] and gene expression [10,11], that promote a proinflammatory state. This response activates within minutes to hours and involves the production of various chemokines, cytokines and angiogenic factors like TNF- $\alpha$ and interleukins as well as nonprotein signals, such as nitric oxide, that are synthesized by resident and recruitable cells [8]. In the central nervous system, innate immune functions are principally carried out by microglia $[12,13]$, although astrocytes [14], endothelial cells [15], neurons $[16,17]$ and in pathological conditions, infiltrating peripheral white blood cells $[18,19]$ may all contribute. As a result of this intercellular cytokine-mediated paracrine and autocrine signaling, the innate response is activated, propagated and amplified, providing both immune defense and tissue repair [2].

There is accumulating evidence that innate neuroinflammatory processes with pathological properties are activated by chronic alcohol exposure [2,20-22]. Yet, how these processes are engaged during withdrawal and their relationship to the resultant anxiety and cardiorespiratory disturbance are poorly understood. The hallmarks of alcohol withdrawal are potentially lifethreatening emotional and autonomic instability characterized by anxiety, agitation, delirium, and sympathetic signs of elevated heart rate and blood pressure [23-27]. These disturbances are anatomically associated with the central nucleus of the amygdala (CeA) and the dorsal vagal complex (DVC), two key viscerosensory nuclei. The former is part of the limbic system and known to be altered in alcohol exposure and withdrawal [28-30]; The latter is a brainstem nucleus that receives afferent from the viscera and moderates vagal influence on cardiac and respiratory functions, also profoundly affected by withdrawal [31]. The CeA receives direct autonomic input from the DVC $[32,33]$, and together they act as part of a viscerosensory and motor circuit to integrate and modulate physical and emotional aspects of autonomic outflow, motivation, affect and emotional learning. Here, we examine the CeA and DVC, specific neural structures associated with the primary symptoms of withdrawal, for evidence of an altered inflammatory state following cessation of the chronic alcohol diet.

It may be expected that removal of alcohol should promote resolution of any proinflammatory state resulting from chronic exposure, leading to recovery. However, there is evidence to suggest that the opposite may occur; repeated cycles of alcohol exposure and withdrawal may exacerbate cellular level oxidative stress and inflammation $[34,35]$. Abrupt changes in the extracellular CNS environment as alcohol levels decrease may directly stress cells, particularly after prolonged exposures leading to molecular adaptation and dependence. By surveying important features of innate immunity during the first 48 hours of alcohol withdrawal in rats following a single period of chronic exposure, here we differentiate between these two possible outcomes. In the CeA, changes consistent with an increased proinflammatory response were identified. Additionally, we identified similar changes in the DVC, suggesting that inflammatory signals are present in these regions anatomically associated with emotional and autonomic instability during early withdrawal.

\section{Methods}

\section{Animals}

Male Sprague Dawley rats $(>120$ g, Harlan, Indianapolis, IN, USA) were housed individually in the Thomas Jefferson University (TJU) Alcohol Research Center Animal Core Facility. Facilities were maintained at constant temperature and humidity with $12 / 12$ hour light cycles. For quantitative reverse transcription polymerase chain reaction (qRT-PCR) studies, animals were assigned to four treatment groups: control, chronic alcohol exposure, four-hour withdrawal or 
forty-eight-hour withdrawal following a thirty-five-day chronic exposure. In immunohistochemistry studies, animals were assigned to three treatment groups: control, chronic alcohol exposure, or 48-hour withdrawal following chronic exposure. In this set of experiments, for technical reasons, animals were used that had received eight months of ethanol or control diet. As in previously published studies from this facility [31] and elsewhere [25], chronic and withdrawal animals were fed the Lieber-DeCarli liquid alcohol diet (36\% of calories as alcohol) ad libitum throughout the exposure period [36,37]. Control rats were fed a liquid diet where alcohol was isocalorically replaced with carbohydrate and diet volume equaled the average consumption of alcohol-fed littermates. No additional water or chow was provided to any of the animals during the study period, ensuring that the animals' entire nutrient and fluid intake came from the alcohol diet. To initiate withdrawal, the alcohol diet was removed at the appropriate time to ensure that all animals would be sacrificed five hours into the light cycle, to account for potential differences due to diurnal factors. All protocols were approved by the TJU Institutional Animal Care and Use Committee.

In the Lieber-DeCarli protocol, blood alcohol levels are not externally controlled during the experiment. Rather, each animal is allowed to self-regulate its oral alcohol intake. Studies using the Lieber-DeCarli method in this facility and elsewhere have shown peak blood alcohol concentrations of 20 to $30 \mathrm{mM}$ with an average daily alcohol intake of 12 to $16 \mathrm{~g} / \mathrm{kg}$ in rats following long-term exposure ( $>3$ weeks) [38-40]. Rats on the full-strength liquid alcohol diet in our facility have comparable intake, as previously published [31]. There were no differences in average intake between the chronic alcohol-exposed and withdrawn animals.

To initiate withdrawal, the alcohol diet was replaced with the control diet. Matched chronically exposed rats were given free access to the alcohol diet until sacrifice. Previous studies and our experience show that symptomatic alcohol withdrawal in rats following a long-term liquid alcohol diet begins within hours and resolves over a two- to three-day period [41-44], though withdrawal symptomatology was not systematically assessed during this study. Studies of alcohol clearance following the cessation of the liquid alcohol diet have shown that clearance rate is approximately linear, and is reduced to less than $25 \%$ of original levels seven hours after removal of the alcohol diet [39]. Similarly, liquid ethanol diet exposures longer than 10 days generate physiologic and behavioral dependence, as evidenced by autonomic and somatic dysfunction upon withdrawal. Within withdrawal's first four hours, these animals show an increased susceptibility to audiogenic convulsions, fragmented sleep, piloerection, tail stiffening, reduced grooming, abnormal gait, reduced motor activity, exaggerated startle, vocalizations and tremors $[40,42]$ that resolve over the first
48 to 72 hours $[40,43]$. While the behavioral and electrographic abnormalities associated with withdrawal from the Lieber-DeCarli diet over time are well characterized, there is little information available about molecular changes in the inflammatory state of the brain during this period. To examine these changes in the central nervous system inflammatory response during this period, we sampled following chronic exposure and at four and forty-eight hours after alcohol removal. Figure 1 shows a schematic of the experimental design.

\section{CeA and DVC microdissection and qRT-PCR}

At the assigned time of sacrifice (four or forty-eight hours after removal of the alcohol diet for withdrawal animals), withdrawn, chronically alcohol-exposed, and match-fed control animals were sacrificed by rapid decapitation and brainstems were excised, placed into icecold artificial cerebral spinal fluid (ACSF: $10 \mathrm{mM}$ HEPES, pH 7.4; $140 \mathrm{mM} \mathrm{NaCl} ; 5 \mathrm{mM} \mathrm{KCl} ; 1 \mathrm{mM}$ $\mathrm{MgCl}_{2} ; 1 \mathrm{mM} \mathrm{CaCl} ; 24 \mathrm{mM}$ D-glucose) and secured with agarose for sectioning (4\% UltraPure ${ }^{\mathrm{Tm}}$ low melting point agarose (Invitrogen, Carlsbad, CA, USA) in ACSF). Transverse sections were made with a Mcllwain tissue chopper (McIlwain, Gamshall, England) for CeA $(625 \mu \mathrm{m})$ and DVC $(275 \mu \mathrm{m})$ microdissection with sizematched micropunches $(\leq 1.25 \mathrm{~mm}$; Stoelting, Wood Dale, IL, USA), as previously reported [45]. Bilateral region punches from one animal were treated as a single sample. Total sample numbers were as follows: CeA Control $\mathrm{N}=10$, chronic ethanol exposure $\mathrm{N}=5$, 4-hour withdrawal $\mathrm{N}=5$, 48-hour withdrawal $\mathrm{N}=3$; NTS Control $=11$, chronic $\mathrm{N}=5$, 4-hour withdrawal $\mathrm{N}=7$, 48hour withdrawal $\mathrm{N}=5$.

Total RNA was extracted with either the RNeasy or the AllPrep DNA/RNA extraction kit (Qiagen, Valencia, CA, USA), DNAase treated (DNA-free RNA kit, Zymo Research, Orange, CA, USA), and stored at $-80^{\circ} \mathrm{C}$. Concentration and integrity were assessed with an ND-1000 (NanoDrop, Wilmington, DE, USA) and RNA Nano 6000 chips on an Agilent 2100 Bioanalyzer. cDNA was reverse transcribed with SuperScript II (Invitrogen) from $100 \mathrm{ng}$ total RNA and stored at $-20^{\circ} \mathrm{C}$.

Intron-spanning PCR primers and probes were designed using the Roche Universal Probe Library Assay Design Center (http://www.universalprobelibrary.com) as indicated: TNF- $\alpha$ forward gtagcccacgtcgtagcaa reverse ggttgtctttgagatccatgc and UPL Probe \#79, Ccl2 (MCP-1) forward agcatccacgtgctgtctc reverse gatcatcttgccagtgaatgag and UPL Probe \#62, NOS-2 forward ggtctttgaaatccctcctga reverse agctcctggaaccactcgta and UPL Probe \#67, the TNF receptor Tnfrsfla forward aatgggggagtgagagagg reverse acccetgatgggtgtatcc and UPL Probe \#21, and the MHC II invariant chain CD74 forward cttccatgtccagtggctct reverse gctgttgtttgaaatgagcaag and UPL Probe \#65. The standard 


\section{A}

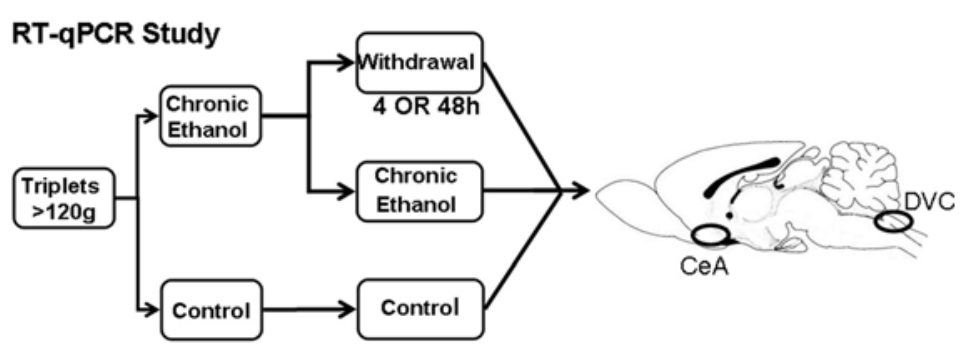

B

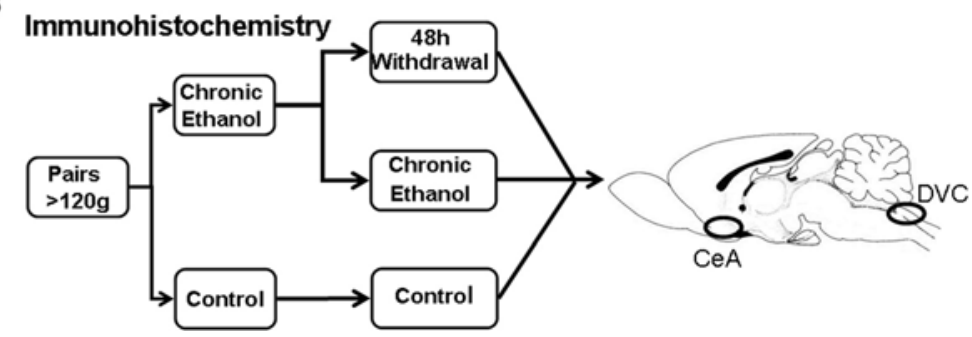

Figure 1 Experimental Design. (A) For the gene expression qRT-PCR study, triplet rats were assigned to control, chronic exposure, and four- or forty-eight-hour withdrawal. Following a 35-day liquid alcohol exposure, animals were sacrificed and the RNA extracted from their CeA and DVC. (B) For IHC study, four pairs of animals were assigned to either the chronic exposure or 48 hours of alcohol withdrawal following eight months on the alcohol diet. Forty-eight hours prior to the time of sacrifice, the withdrawal animals had their diets removed to initiate withdrawal. Following intracardiac perfusion, both rats were sacrificed and their CeA and DVC collected for immunohistochemistry and confocal microscopy. CeA, central nucleus of the amygdala; DVC, dorsal vagal complex; qRT-PCR, quantitative reverse transcription polymerase chain reaction; IHC, immunohistochemistry.

BioMark $^{\text {Tix }}$ protocol was used to preamplify cDNA samples for 16 cycles using TaqMan PreAmp Master Mix per the manufacturer's protocol (Applied Biosystems, Foster City, CA, USA). qPCR reactions were performed using BioMark $^{\text {Tm }}$ 96.96 Dynamic Arrays (Fluidigm, South San Francisco, CA, USA) enabling quantitative measurement of multiple mRNAs and samples under identical reaction conditions $[46,47]$. Runs were forty cycles (fifteen seconds at $95^{\circ} \mathrm{C}$, five seconds at $70^{\circ} \mathrm{C}$, sixty seconds at $60^{\circ} \mathrm{C}$ ). $\mathrm{C}_{\mathrm{T}}$ values were calculated by the Real-Time PCR Analysis software (Fluidigm) and software-designated failed reactions were discarded from the analysis.

\section{qRT-PCR data normalization and analysis}

Normalization $\left(\Delta C_{T}\right)$ to the average expression of the housekeeping genes $A c t b$ and Rpl13a was performed using the $\mathrm{R}$ statistical computing package [48-50]. Two-way analysis of variance (ANOVA) performed on $\Delta C_{T}$ values was used to identify genes with a significant region or treatment effect. ANOVA identifies the variability in data that is associated with and potentially attributable to each experimental factor (treatment and region). Post hoc significance testing was performed using a Tukey's honestly significant difference (HSD) test to identify pair-wise differences between treatment times. Here, a region ANOVA $P$ value of less than 0.05 indicates a significant difference in mRNA levels in the CeA and DVC, while a treatment ANOVA $P$ value of less than 0.05 indicates a significant cumulative effect on mRNA levels over the four treatment conditions: control, chronic alcohol exposure and fourhour and forty-eight-hour withdrawal. Tukey's HSD values of less than 0.05 identify pair-wise differences between individual treatment conditions. Mean differences in mRNA expression between treatment groups are expressed as $\Delta \Delta C_{T}$ values by calculating the average $\Delta C_{T}$ values of each gene at a given treatment time and subtracting this value from the mean $\Delta \mathrm{C}_{\mathrm{T}}$ of all controls [51]. In this way, a $\Delta \Delta \mathrm{CT}=1$ corresponds to a 2 -fold increase in expression. All statistical tests were conducted at a $95 \%$ confidence level $(P \leq 0.05)$.

\section{Intracardiac perfusion, immunohistochemistry and confocal microscopy}

For immunohistochemistry (IHC), on the assigned day of sacrifice, animals were anesthetized by placing the rat in an induction chamber preloaded with isoflurane (5\% in oxygen). After induction, $2 \%$ isoflurane was used for maintenance during PBS intracardiac perfusion with $50 \mathrm{~mL}$ of phosphate buffered saline (PBS, $\mathrm{pH}$ 7.2) The rats were then sacrificed by rapid decapitation, the 
brains quickly extracted and placed in ice cold ACSF. The brain-stem and forebrain were dissected and frozen separately in optimal cutting temperature (OCT) and stored at $-80^{\circ} \mathrm{C}$ until cryostat slicing.

The embedded forebrain and brainstem blocks were sectioned in a cryostat at $10 \mu \mathrm{m}$ thickness, and thaw mounted on glass slides. Slides containing the neuroanatomically identified DVC and CeA regions were first fixed in $100 \%$ cold methanol for five minutes, then briefly rinsed in PBS three times, five minutes each. Sections were then blocked and permeabilized with $\mathrm{PBS}$ containing $2 \%$ bovine serum albumin (BSA) and $0.1 \%$ Triton X-100 (Sigma-Aldrich, St. Louis, MO, USA) for one hour. Afterwards, brain sections were incubated with the primary antibody (see below) overnight at $4{ }^{\circ} \mathrm{C}$. Then slides were washed and incubated for two hours at room temperature in the dark with the secondary antibody (see below). Finally, slides were washed, mounted with Fluorsave ${ }^{\mathrm{Tw}}$ (Calbiochem, San Diego, CA, USA) and stored at $4^{\circ} \mathrm{C}$ in the dark. Staining controls were performed by incubating with PBS instead of the primary antibody or both antibodies (data not shown).

In order to visualize neurons, mouse monoclonal anti-NeuN (Millipore, Billerica, MA, USA; dilution 1:1000 in PBS-BSA) was used. Alexa-555 goat antimouse immunoglobulin G (IgG) (Jackson ImmunoResearch Laboratories, West Grove, PA, USA; dilution 1:1000 in PBS-BSA) was used as the secondary antibody to detect anti-NeuN staining. TNF- $\alpha$ protein was visualized with polyclonal rabbit anti-TNF- $\alpha$ (eBioscience, Glostrup, Denmark; dilution 1:100 in PBS-BSA) and Alexa-488 goat anti-rabbit IgG (Jackson; dilution 1: 1000 in PBS-BSA). Mouse monoclonal anti-rat endothelial cell antigen-1 (RECA-1) (Genway Biotechnology, San Diego, CA, USA diluted 1:10 in PBS-BSA) was used as the primary antibody to localize blood vessels [52]. RECA-1 staining identifies terminal arterioles, arterial capillaries and venous capillaries, postcapillary-sized venules, and collecting venuoles $[53,54]$. In the hippocampus, arterial microvessels and capillaries were shown to stain more strongly then venous vessels. While there are differences at the anatomic and molecular levels between microvessels [55], they were not subclassified in this study. Alexa-555 donkey anti-mouse IgG (Invitrogen; dilution 1:1000 in PBS-BSA) or Alexa633 donkey anti-mouse (Invitrogen; dilution 1:1000 in PBS-BSA) was used as the secondary antibody to detect anti-RECA-1 staining. To detect the presence of intercellular adhesion molecule 1 (ICAM-1), rabbit anti-rat CD54 monoclonal antibody (Millipore; dilution 1:100 in PBS-BSA) was used with Alexa-488 donkey anti-rabbit IgG (Invitrogen; 1:1000 in PBSBSA). Finally, for nuclei staining, the slides were washed with PBS, and then incubated with $5 \mu \mathrm{g} / \mathrm{ml}$ 4'-6-diamidino-2-phenylindole (DAPI) (SigmaAldrich) at room temperature for five minutes.

\section{Confocal microscopy}

Confocal microscopy (Zeiss 510 Meta, Göttingen, Germany) was performed with an x63 objective lens (oil, numeric opening 1.4). We used an argon laser (excitation 488, emission 505 to $530 \mathrm{~nm}$ ) for Alexa-488, a helium laser (excitation 543, emission 585 to $615 \mathrm{~nm}$ ) for Texas Red and a krypton-argon laser (excitation $647 \mathrm{~nm}$, emission 660 to $700 \mathrm{~nm}$ ) for Alexa-647. Images were collected sequentially to avoid cross-contamination between fluorochromes. A series of 15 optical sections was projected onto a single image plane and scanned at $1024 \times 1024$ pixel resolution. Images are pseudocolored for visualization.

\section{Results}

\section{CCl2, NOS-2, TNF- $a$, Tnfrsf1 $a$ and CD74 gene expression during alcohol withdrawal}

The objective of this study was to identify changes in innate immunity in the CNS during the first 48 hours of alcohol withdrawal following a single long-term exposure in two regions anatomically associated with the emotional and autonomic instability associated with alcohol withdrawal. We examined the gene expression of Ccl2, NOS-2, TNF- $\alpha$, Tnfrsf1a and CD74 as surrogate markers of inflammation in the CeA of adult male rats exposed to a liquid alcohol diet for thirty-five days and in similarly treated animals four hours and forty-eight hours into alcohol withdrawal. The CeA is an alcohol-responsive part of the limbic system, thought to play a major role in drug addiction and the coordination of autonomic and emotional behaviors [28]. We took additional confirmatory measures in a second directly connected brain region [33], the alcohol responsive DVC, also relevant to physiologic sympathetic response during alcohol withdrawal [31].

TNF- $\alpha\left(P=2.85 \times 10^{-4}\right), N O S-2(P=0.005), C c l 2(M C P-$ $1, P=0.041)$, MHC II invariant chain CD74 $(P=0.007)$, and the TNF receptor Tnfrsfla $(P=0.041)$ all showed statistically significant cumulative treatment effects identified by analysis of variance (ANOVA), as shown in Figure 2 . While we also noted significant regional expression differences in TNF- $\alpha\left(P=7.32 \times 10^{-4}\right)$ and $C c l 2\left(P=1.73 \times 10^{-5}\right)$, there were no significant region-treatment interactions. Figure 2A shows the increase in CeA mRNA levels of TNF$\alpha, N O S-2, C c l 2$ and $C D 74$ during withdrawal. Further examination shows that these changes are largely attributable to increases measured at 48 hours, reaching a near doubling $\left(\Delta \Delta \mathrm{C}_{\mathrm{T}}=1\right)$ of control levels, while chronic alcohol exposure resulted in relatively small increases in expression. Follow-up pair-wise post hoc Tukey's HSD testing to identify differences between individual time points was then 

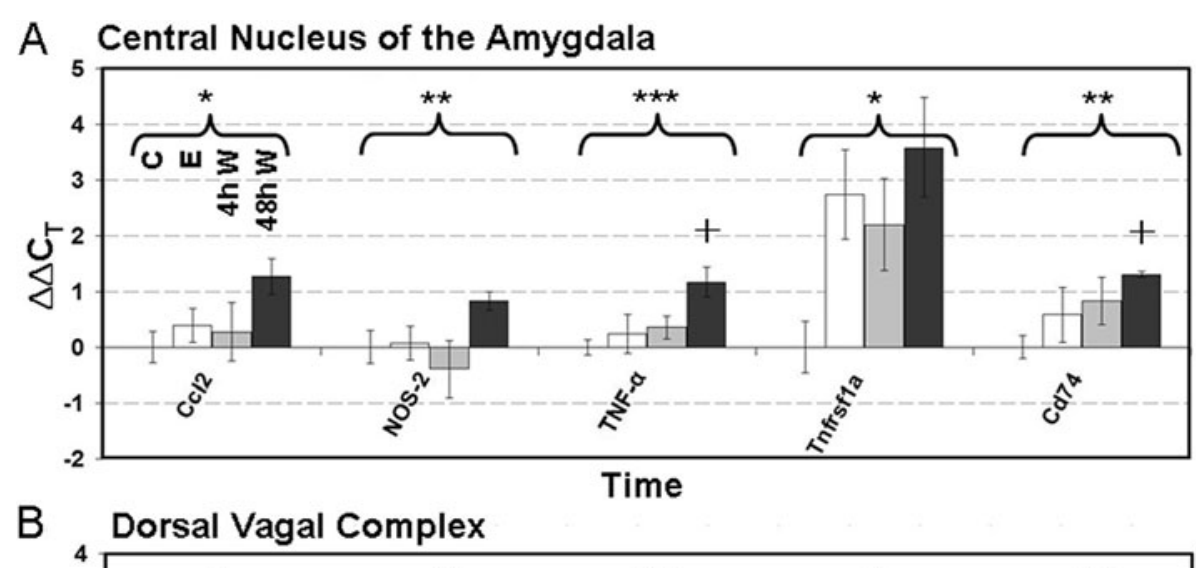

Time

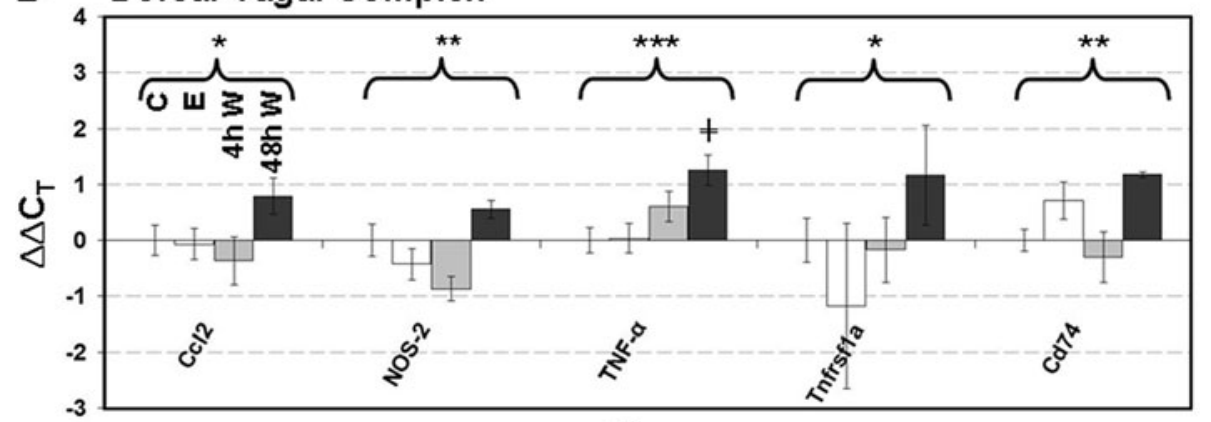

Time

Figure 2 qRT-PCR analysis of expression of CCl2, NOS-2, TNF- $a$, Tnfrsf1a and CD74 during the first 48 hours of alcohol withdrawal. Alcohol-treated rats were fed the Lieber-DeCarli liquid alcohol diet for 35 days prior to forced withdrawal. qRT-PCR was performed on CeA and DVC samples. All transcripts were found to have a significant treatment effect by two-factor ANOVA, and to change significantly as a result of alcohol treatment and withdrawal. (A) CeA mean $\Delta \Delta C_{T}$ values for the transcripts CCI2, NOS-2, TNF- $a$ and Tnfrsfia and CD74 in control (C; $\left.N=10\right)$, chronically alcohol exposed ( $\left.E_{;} N=5\right)$, and 4 hours $(4 \mathrm{~h} \mathrm{~W} ; \mathrm{N}=5)$ and 48 hours ( $48 \mathrm{~h} \mathrm{~W}, \mathrm{~N}=3$ ) withdrawn rats. (B) DVC mean $\triangle \triangle C_{T}$ values $(C \mathrm{~N}=$ $11 ; E=5,4 h W n=7,48 h N=5)$. $A \Delta \Delta C_{T}=1$ is a doubling of control mRNA levels. Error bars represent the $+/-S E M$. *Significant treatment effect identified via two-way ANOVA $(P \leq 0.05)$; **Significant treatment effect identified via two-way ANOVA $(P \leq 0.01)$; ** Significant treatment effect identified via two-way ANOVA ( $P \leq 0.005)$; † Significant post hoc Tukey's test versus control, $(P \leq 0.01$.) ANOVA, two-way analysis of variance; $C \subset$, chemokine (C-C motif) ligand 12; CeA, central nucleus of the amygdala; DVC, dorsal vagal complex; NOS-2, inducible nitric oxide synthase; qRT$\mathrm{PCR}$, quantitative reverse transcription polymerase chain reaction.

performed to compare individual treatment conditions to one another. Time-point-specific testing showed that at 48 hours changes in both TNF- $\alpha$ and CD74 mRNA were significantly different from control values by post hoc Tukey's testing $(P \leq 0.05)$. Tnfrsfla expression in the CeA was also affected by alcohol exposure and withdrawal, though its pattern was distinct from the other transcripts. The TNF- $\alpha$ receptor mRNA was strongly upregulated during chronic alcohol exposure, with a nonsignificant trend toward an additional increase during withdrawal.

Figure $2 \mathrm{~B}$ shows the analogous gene expression in the DVC. Again for Ccl2, NOS-2, TNF- $\alpha$, Tnfrsfla and CD74, we saw a significant mRNA induction over the first 48 hours of withdrawal reaching an approximate doubling of control mRNA levels. As in the CeA, Tukey's testing identified a significant difference between 48-hour TNF- $\alpha$ levels in comparison to control $(P<0.01)$. Additionally, a statistically significant difference was found in the DVC expression levels of CD74 between four and forty-eight hours of withdrawal $(P \leq 0.05)$. Similar to the chronic changes seen in the CeA, DVC samples taken during prolonged exposure also showed relatively small changes in mRNA levels of Ccl2, NOS-2, TNF- $\alpha$ and CD74. However, DVC levels of these transcripts were mildly reduced rather than upregulated in chronically exposed animals, though these effects were nonsignificant by post hoc testing. Again, Tnfrsla showed a unique expression pattern, with a large average decrease during chronic exposure.

\section{Localization of TNF- $a$, RECA-1 and ICAM-1 expression}

As TNF- $\alpha$ is known to be a central provocateur of inflammation, we sought to confirm the presence of the TNF- $\alpha$ protein in alcohol-withdrawn animals as a marker of active early inflammation, and to compare IHC 
staining of CeA in the control condition, and in similarly treated animals with an eight-month chronic alcohol exposure and forty-eight-hour withdrawal. In this way, the IHC images serve as a qualitative confirmation of the presence of an innate immune response during alcohol withdrawal as suggested by our quantitative PCR measures. Representative images of the staining in these three conditions are shown in Figure 3A to C. Consistent with the normal noninflamed state of the brain, the control CeA samples showed only sparse TNF- $\alpha$ staining. Samples taken during chronic alcohol exposure showed more pronounced TNF- $\alpha$ staining, demonstrating persistent elevations after an eight-month exposure, consistent with our qPCR measures. CeA 48-hour withdrawal samples showed increased TNF- $\alpha$ staining in comparison to both control and chronic samples, confirming the presence of a protein-level inflammatory response. Additionally, CeA withdrawal samples showed dual labeling of TNF- $\alpha$ and NeuN, suggesting neuronal production of the cytokine. This differs from the control and chronic conditions where CeA TNF- $\alpha$ staining appears to be localized to discrete areas surrounding cells expressing the neural marker NeuN, as shown in Figure $3 \mathrm{~B}$ to $\mathrm{C}$. Similar, but less pronounced findings were observed in the DVC, with increased TNF- $\alpha$ staining in the chronic and withdrawal conditions. However, no localization differences were observed in the DVC (Figure 3D to F), and intracellular TNF- $\alpha$ staining can be seen in all treatments, including in the control DVC neuron in Figure 3D.

To determine if neurovascular endothelial cells undergo a response consistent with inflammatory activation, we performed IHC for ICAM-1 (CD54), a cellular adhesion molecule upregulated by TNF- $\alpha$ [56-58]. Control CeA samples showed limited RECA-1 endothelial staining with no ICAM-1 colabeling, consistent with normal vascular endothelia (Figure 3G). However, following the eight-month alcohol exposure, RECA-1 and ICAM-1 colabeling became apparent (Figure $3 \mathrm{H})$. This colabeling was also present in the 48-hour withdrawal condition, confirming the presence of endothelial cells expressing cellular adhesion molecules during withdrawal. Again, these findings were confirmed in the DVC as shown in Figure 3K to L. Finally, during exposure and withdrawal there is considerable ICAM-1 expression in the cells surrounding the vasculature in both the CeA and DVC (Figure $3 \mathrm{H}$ to I, K to L).

\section{Discussion}

This study demonstrates in vivo that alcohol withdrawal following a single long-term alcohol exposure is associated with increased inflammation. We confirmed this observation in two brain regions neuroanatomically associated with emotional and autonomic regulation that are notably disrupted during withdrawal: the central nucleus of the amygdala (CeA) and the dorsal vagal complex (DVC). Our study demonstrates the increased expression of mRNAs specific for several inflammatory markers in these regions including the inflammatory cytokine $T N F-\alpha$ and its receptor Tnfrsfla, monocyte chemoattractant $\mathrm{Ccl} 2$, inducible nitric oxide synthase NOS-2, and the major histocompatability complex class II antigen (MHC II) invariant chain CD74 over a 48-hour withdrawal. By demonstrating IHC staining for TNF- $\alpha$ surrounding NeuN-positive neurons and ICAM-1 in RECA-1-expressing endothelia, we also verified the presence of an inflammatory response at the protein level. Additionally, consistent with other previously reported studies, we observed a mild inflammatory state associated with chronic intake. These qRT-PCR and IHC studies indicate that the first 48 hours of alcohol withdrawal are characterized by an exacerbation of alcohol-induced proinflammatory changes in brain regions anatomically associated with the maintenance of emotional and cardiorespiratory homeostasis which are known to be disrupted during withdrawal in humans $[24,59]$ and animal models $[27,31]$ over the same time frame.

The protein products of the TNF- $\alpha$ and Tnfrsfla genes act together to activate and amplify the inflammatory response, and increased expression during alcohol withdrawal suggests that these processes are activated. NOS2 encodes inducible nitric oxide synthase, suggestive of concurrent oxidative stress. The concomitant increases in CD74 and Ccl2 may indicate that changes in immune cell composition are occurring, potentially altering the number, activation state or type of antigen-presenting cells in these nuclei during withdrawal. These findings may have important consequences on emotional and cardiorespiratory regulation, as CNS inflammation has been shown to alter physiology and behavior. For example, injection of TNF- $\alpha$ into the ventricles causes elevations in mean arterial pressure [60] and repeated microinjections of TNF- $\alpha$ and Ccl2 into the amygdala prior to prolonged alcohol exposure have been associated with an exaggerated anxiety-like response during withdrawal $[22,61]$. Similarly, peripheral lipopolysaccharide (LPS) injection in rats has also been shown to amplify anxiety-like withdrawal behavior [22]. Conversely, TLR4 - /- knockout mice that fail to express a receptor critical to the innate immune response have been shown to be resistant to behavioral and cognitive changes associated with alcohol exposure. Specifically, TLR4 -/knockout mice show neither the decreased exploratory activity eight hours into withdrawal following a five-month alcohol exposure nor the impaired cognitive testing following a fifteen-day withdrawal that is typical in the wild-type [62]. This suggests that these changes are dependent on an intact innate inflammatory response. Our studies support this conclusion by directly measuring increases in several innate immune signals within the first 48 hours of 


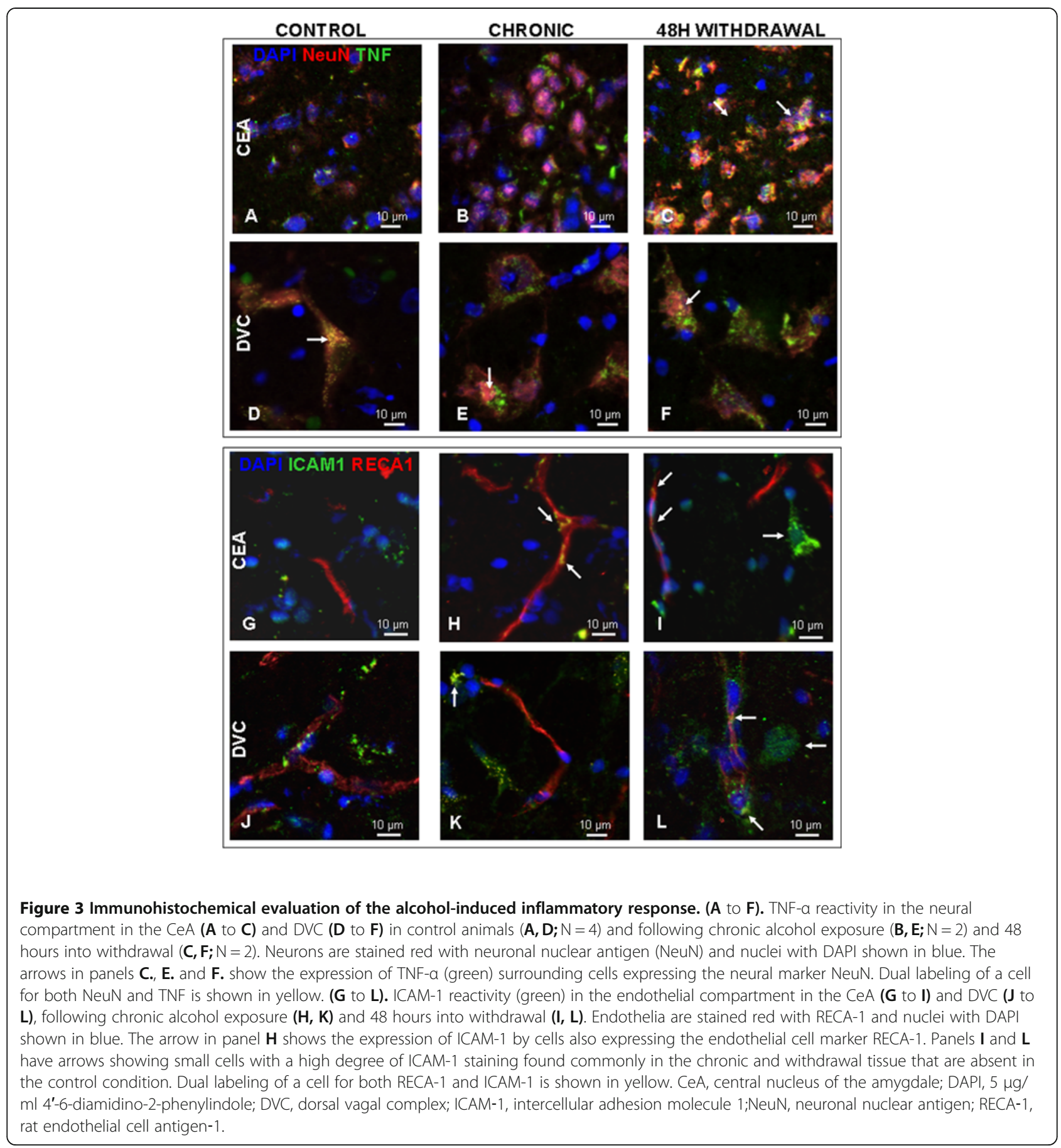

withdrawal, demonstrating an innate immune response as a primary element of withdrawal pathology. Consequently, repeated cycles of inflammation induced by CeA TNF- $\alpha$ microinjection as in the Knapp Study [22], or synthesized endogenously as a consequence of repeated episodes of exposure and withdrawal, may worsen withdrawal symptoms by amplifying this innate immune response.

Notably, inflammation in the CeA and DVC, brain regions that act to regulate emotional and physiologic homeostasis, could form a considerable barrier to abstinence and contribute to the cycle of negative reinforcement that sustains dependent drinking behaviors [63]. The therapeutic implications of this finding are important, suggesting that immunomodulators may be effective in treating emotional and autonomic dysregulation during withdrawal. Additionally, the involvement of embryologically, neuroanatomically and functionally diverse DVC and CeA brain regions 
raises questions of the generalizability of this active innate immune response in other brain regions. Further, the demonstration of a short-term innate immune response during withdrawal raises questions about the relationship between alcohol-related neurodegeneration and withdrawal rather than consumption in isolation. However, studies that confirm the presence of a sustained inflammatory response in these and other brain regions classically associated with alcohol-related neurodegeneration including the cerebellum, hippocampus, entorhinal and perirhinal cortices [34,35,64-66] along with direct assessments of neural injury comparing prolonged exposure with and without withdrawal periods are necessary, to further explore this hypothesis.

Earlier work has focused on the inflammatory consequences of long-term alcohol exposure, yet few studies have characterized these processes in withdrawal directly. Studies of neurons and glia exposed to alcohol in culture have shown a variable response, including increases in the generation of reactive oxygen species, prostaglandins [14], and NFkB DNA-binding [21] that has been purported to be both injurious to and protective of central nervous system cells [67]. In vivo studies have worked to clarify these seemingly contradictory findings, and suggest that these consequences are largely proinflammatory and injurious; following long-term alcohol exposure, murine frontal cortex samples have increased expression of NOS-2, ionized calcium binding adaptor molecule 1 (Iba1), and 3-nitrotyrosine protein adduct levels consistent with tissue injury [68], and demonstrate sustained increases in the production of TNF- $\alpha$, MCP- 1 and Il- $1 \beta$ in the brain following intraperitoneal LPS injection without direct CNS injury, confirming a proinflammatory state [4]. While direct studies of withdrawal are limited, Brown and colleagues showed that in hippocampalentorhinal cortical slice cultures, repeated cycles of exposure and withdrawal led to increased neural damage that could be partially inhibited by treatment with the PLA2-inhibitor mepacrine and the anti-inflammatory lipid docosahexaenoic acid [35]. In contrast, a single four-day exposure of alcohol and twenty-four-hour withdrawal period was not associated with increased TNF- $\alpha$, IFN-Y, Il-1b, Il-4, Il-5, Il-13 or Cxcl1 protein levels in the brain of rats [69]. Here, our measures suggest that a single longterm alcohol exposure followed by a 48-hour withdrawal is sufficient to induce a significant central nervous system inflammatory response, larger in magnitude than that associated with chronic exposure. Other work from our laboratory examining early withdrawal transcription dynamics in the DVC [31] and CeA (in review) showed surprisingly large and extensive transcriptional responses suggestive of profound changes in intracellular signaling, potentially consistent with active inflammation. Thus, this study aims to follow up these results with the focus on neuroimmune processes. Its results, most notably the increases in $T N F-\alpha$, strengthened by concomitant increases in the mRNA expression of Ccl2, NOS-2, Tnfrsf1a and CD74, provide more direct evidence of a neuroimmune response in these nuclei anatomically related to withdrawal's emotional and homeostatic imbalance.

\section{Conclusion}

In summary, the current findings show that alcohol withdrawal induces an acute exacerbation of the limited proinflammatory response seen during long-term chronic alcohol exposure at both the mRNA and protein level at 48 hours. Our in vivo measurement of increases in TNF- $\alpha$, Tnfrsfla, Ccl2, NOS-2 and CD74 during withdrawal suggests that the period can be viewed as an acute-on-chronic inflammatory process, where proinflammatory changes that occur during chronic alcohol exposure are worsened immediately following the removal of alcohol from the cellular environment. As a consequence of the roles of these regions in emotional and physiologic regulation, these findings may have important implications for the treatment of withdrawal-related autonomic and emotional dysfunction. Targeted investigations aimed at characterizing cell-specific interactions in neurons and glia, as well as studies aimed at pharmacologic manipulation of these central inflammatory pathways in these homeostatic brain regions may increase our understanding of withdrawal and its associated affective and cardiorespiratory effects.

\section{Abbreviations}

ANOVA: two-way analysis of variance; BSA: bovine serum albumin; CCl2: chemokine (C-C motif) ligand 2; CeA: central nucleus of the amygdala;

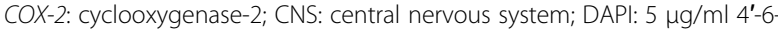
diamidino-2-phenylindole; DVC: dorsal vagal complex; Iba-1: ionized calcium binding adaptor molecule 1; ICAM-1: intercellular adhesion molecule 1; Ig: immunoglobulin; IHC: immunohistochemistry; IL: interleukin; MCP1: monocyte chemotactic protein-1; HSD: honestly significant difference; LPS: lipopolysaccharide; MCH-II: major histocompatability complex class II antigen; NeuN: neuronal nuclear antigen; NOS-2: inducible nitric oxide synthase; OCT: optimal cutting temperature; PBS: phosphate buffered saline; qRT-PCR: quantitative reverse transcription polymerase chain reaction; RECA1: rat endothelial cell antigen-1; TNF-a: tumor necrosis factor-alpha.

\section{Competing interests}

The authors declare that they have no competing interests.

\section{Acknowledgements}

This work was supported by grants from the National Institutes of Health $(\mathrm{NIH})$ (R01 AA-015601, R01 GM-076495 and R33 HL-087361 to JSS; GM-083108 to JSS and RV; R33 HL088283 to RV; R24 AA-014986 to JBH; and T32 AA-007463 support of KF and MMS). The qRT-PCR was performed with generous help from Carmen N. Nichols, PhD and Haisun Zhu, PhD. We also wish to thank Prudhvi Mummaneni and James Park for their assistance with analysis and the TJU Alcohol Research Center, especially Dr. Biddanda Ponnappa, John Mullen, and Permelia Mullen, for their support with the animal model.

\section{Author details}

'Daniel Baugh Institute for Functional Genomics and Computational Biology, Department of Pathology, Anatomy and Cell Biology, Thomas Jefferson University, 1020 Locust Street, Philadelphia, PA 19107, USA. ${ }^{2}$ Department of 
Chemical Engineering, Rowan University, 201 Mullica Hill Road, Glassboro, NJ 08028, USA. ${ }^{3}$ Department of Cancer Biology and Neurosurgery, Thomas Jefferson University, 111 South 11th Street, Philadelphia, PA 19107, USA.

\section{Authors' contributions}

KF conceived of the study, carried out the qRT-PCR, performed the statistical analysis and drafted the manuscript. AB participated in the experimental design and carried out the GRT-PCR and IHC studies with MMB. RV directed the data normalization and statistical analysis. MMS participated in the experimental design, and performed the animal experiments for the GRTPCR. GEG, JBH, DCH and JSS contributed specific subject matter expertise in molecular biology, animal models of alcohol exposure, neuroscience and neuroinflammation. All authors read and approved the final manuscript.

Received: 8 February 2012 Accepted: 24 May 2012

Published: 24 May 2012

\section{References}

1. Kelley KW, Dantzer R: Alcoholism and inflammation: neuroimmunology of behavioral and mood disorders. Brain Behav Immun 2011, 25(Suppl 1):S13-S20

2. Crews FT, Zou J, Qin L: Induction of innate immune genes in brain create the neurobiology of addiction. Brain Behav Immun 2011, 25(Suppl 1):S4-S12.

3. Valles SL, Blanco AM, Pascual M, Guerri C: Chronic ethanol treatment enhances inflammatory mediators and cell death in the brain and in astrocytes. Brain Pathol 2004, 4:365-371.

4. Qin L, He J, Hanes RN, Pluzarev O, Hong JS, Crews FT: Increased systemic and brain cytokine production and neuroinflammation by endotoxin following ethanol treatment. J Neuroinflammation 2008, 5:10.

5. Fernandez-Lizarbe S, Pascual M, Guerri C: Critical role of TLR4 response in the activation of microglia induced by ethanol. J Immuno/ 2009, 7:4733-4744.

6. He J, Crews FT: Increased MCP-1 and microglia in various regions of the human alcoholic brain. Exp Neurol 2008, 2:349-358.

7. Yakovleva T, Bazov I, Watanabe H, Hauser KF, Bakalkin G: Transcriptional control of maladaptive and protective responses in alcoholics: a role of the NF-kB system. Brain Behav Immun 2011, 25(Suppl 1):S29-S38.

8. Gorina R, Font-Nieves M, Marquez-Kisinousky L, Santalucia T, Planas AM: Astrocyte TLR4 activation induces a proinflammatory environment through the interplay between MyD88-dependent NFkappaB signaling, MAPK, and Jak1/Stat1 pathways. Glia 2011, 2:242-255.

9. Okun E, Griffioen KJ, Mattson MP: Toll-like receptor signaling in neural plasticity and disease. Trends Neurosci 2011, 5:269-281.

10. Barber GN: Cytoplasmic DNA innate immune pathways. Immunol Rev 2011, 1:99-108.

11. Veroni C, Gabriele L, Canini I, Castiello L, Coccia E, Remoli ME, ColumbaCabezas S, Arico E, Aloisi F, Agresti C: Activation of TNF receptor 2 in microglia promotes induction of anti-inflammatory pathways. Mol Cell Neurosci 2010, 3:234-244.

12. Kofler J, Wiley CA: Microglia: key innate immune cells of the brain. Toxicol Pathol 2011, 1:103-114.

13. Sosa RA, Forsthuber TG: The critical role of antigen-presentation-induced cytokine crosstalk in the central nervous system in multiple sclerosis and experimental autoimmune encephalomyelitis. J Interferon Cytokine Res 2011, 31:753-768.

14. Floreani NA, Rump TJ, Muneer PM, Alikunju S, Morsey BM, Brodie MR, Persidsky $Y$, Haorah J: Alcohol-induced interactive phosphorylation of src and toll-like receptor regulates the secretion of inflammatory mediators by human astrocytes. J Neuroimmune Pharmacol 2010, 4:533-545.

15. Haorah J, Knipe B, Leibhart J, Ghorpade A, Persidsky Y: Alcohol-induced oxidative stress in brain endothelial cells causes blood-brain barrier dysfunction. J Leukoc Biol 2005, 6:1223-1232.

16. Yu CH, Yhee JY, Kim JH, Im KS, Kim NH, Jung DI, Lee HC, Chon SK, Sur JH: Pro- and anti-inflammatory cytokine expression and histopathological characteristics in canine brain with traumatic brain injury. J Vet Sci 2011, 3:299-301.

17. Lisak RP, Nedelkoska L, Studzinski D, Bealmear B, Xu W, Benjamins JA: Cytokines regulate neuronal gene expression: differential effects of Th1, Th2 and monocyte/macrophage cytokines. J Neuroimmunol 2011, 1-2:19-33.

18. Kassner SS, Kollmar R, Bonaterra GA, Hildebrandt W, Schwab S, Kinscherf R: The early immunological response to acute ischemic stroke: differential gene expression in subpopulations of mononuclear cells. Neuroscience 2009, 2:394-401.
19. Thibeault I, Laflamme N, Rivest S: Regulation of the gene encoding the monocyte chemoattractant protein 1 (MCP-1) in the mouse and rat brain in response to circulating LPS and proinflammatory cytokines. $J$ Comp Neurol 2001, 4:461-477.

20. Zou J, Crews F: Induction of innate immune gene expression cascades in brain slice cultures by ethanol: key role of NF-kappaB and proinflammatory cytokines. Alcohol Clin Exp Res 2010, 5:777-789.

21. Zou J, Crews F: CREB and NF-kappaB transcription factors regulate sensitivity to excitotoxic and oxidative stress induced neuronal cell death. Cell Mol Neurobiol 2006, 4-6:385-405.

22. Breese GR, Knapp DJ, Overstreet DH, Navarro M, Wills TA, Angel RA: Repeated lipopolysaccharide (LPS) or cytokine treatments sensitize ethanol withdrawal-induced anxiety-like behavior. Neuropsychopharmacology 2008, 4:867-876.

23. McKeon A, Frye M, Delanty N: The alcohol withdrawal syndrome. J Neurol Neurosurg Psychiatry 2008, 8:854-862.

24. Eyer F, Schuster T, Felgenhauer N, Pfab R, Strubel T, Saugel B, Zilker T: Risk assessment of moderate to severe alcohol withdrawal-predictors for seizures and delirium tremens in the course of withdrawal. Alcohol Alcohol 2011, 4:427-433.

25. Jochum T, Reinhard M, Boettger MK, Piater M, Bar KJ: Impaired cerebral autoregulation during acute alcohol withdrawal. Drug Alcohol Depend 2010, 3:240-246.

26. Monte R, Rabunal R, Casariego E, Lopez-Agreda H, Mateos A, Pertega S: Analysis of the factors determining survival of alcoholic withdrawal syndrome patients in a general hospital. Alcohol Alcohol 2010, 2:151-158.

27. Shirafuji S, Liu J, Okamura N, Hamada K, Fujimiya T: QT interval dispersion and cardiac sympathovagal balance shift in rats with acute ethanol withdrawal. Alcohol Clin Exp Res 2010, 2:223-230.

28. McBride WJ: Central nucleus of the amygdala and the effects of alcohol and alcohol-drinking behavior in rodents. Pharmacol Biochem Behav 2002, 3:509-515

29. Rodd ZA, Kimpel MW, Edenberg HJ, Bell RL, Strother WN, McClintick JN, Carr $L G$, Liang T, McBride WJ: Differential gene expression in the nucleus accumbens with ethanol self-administration in inbred alcohol-preferring rats. Pharmacol Biochem Behav 2008, 4:481-498.

30. McBride K, WJ KMW, Schultz J, McMlintick J, Edenberg H, Bell R: Changes in gene expression in regions of the extended amygdala of alcoholpreferring rats after binge-like alcohol drinking. Alcohol 2010, 2:171183.

31. Freeman K, Staehle M, Gümüş Z, Vadigepalli R, Gonye G, Nichols CN, Ogunnaike B, Hoek J, Schwaber J: Rapid temporal changes in the expression of a set of neuromodulatory genes during alcohol withdrawal in the dorsal vagal complex: molecular evidence of homeostatic disturbance. Alcohol Clin Exp Res 2012. doi:10.1111/j.15300277.2012.01791.x. [Epub ahead of print.

32. Schwaber JS, Kapp BS, Higgins G: The origin and extent of direct amygdala projections to the region of the dorsal motor nucleus of the vagus and the nucleus of the solitary tract. Neurosci Lett 1980, 1:15-20.

33. Schwaber J, Kapp B, Higgins G, Rapp P: Amygdaloid and basal forebrain direct connections with the nucleus of the solitary tract and the dorsal motor nucleus. J Neurosci 1982, 10:1424-1438.

34. Collins MA, Neafsey EJ: Neuroinflammatory pathways in binge alcohol-induced neuronal degeneration: oxidative stress cascade involving aquaporin, brain edema, and phospholipase A2 activation. Neurotox Res 2012, 1:70-78.

35. Brown J, Achille N, Neafsey EJ, Collins MA: Binge ethanol-induced neurodegeneration in rat organotypic brain slice cultures: effects of PLA2 inhibitor mepacrine and docosahexaenoic acid (DHA). Neurochem Res 2009, 2:260-267.

36. Lieber CS, DeCarli LM: An experimental model of alcohol feeding and liver injury in the baboon. J Med Primatol 1974, 3:153-163.

37. Hall Pd, Lieber CS, DeCarli LM, French SW, Lindros KO, Jarvelainen H, Bode C, Parlesak A, Bode JC: Models of alcoholic liver disease in rodents: critical evaluation. Alcohol Clin Exp Res 2001, 25(Suppl 5):254S-261S

38. Lieber CS, DeCarli LM: Animal models of chronic ethanol toxicity. Methods Enzymol 1994, 233:585-594.

39. Wilson JS, Korsten MA, Lieber CS: The combined effects of protein deficiency and chronic ethanol administration on rat ethanol metabolism. Hepatology 1986, 5:823-829.

40. Macey DJ, Schulteis G, Heinrichs SC, Koob GF: Time-dependent quantifiable withdrawal from ethanol in the rat: effect of method of dependence induction. Alcohol 1996, 2:163-170. 
41. Walker DW, Hunter BE, Riley J: A behavioral and electrophysiological analysis of ethanol dependence in the rat. Adv Exp Med Biol 1975, 59:353-372.

42. Hunter BE, Riley JN, Walker DW: Ethanol dependence in the rat: a parametric analysis. Pharmacol Biochem Behav 1975, 4:619-629.

43. Geisler RF, Hunter BE, Walker DW: Ethanol dependence in the rat: temporal changes in neuroexcitability following withdrawal. Psychopharmacology (Berl) 1978, 3:287-292.

44. Macey DJ, Schulteis G, Heinrichs SC, Koob GF: Time-dependent quantifiable withdrawal from ethanol in the rat: effect of method of dependence induction. Alcohol 1996, 2:163-170.

45. Khan RL, Vadigepalli R, McDonald MK, Rogers RF, Gao GR, Schwaber JS: Dynamic transcriptomic response to acute hypertension in the nucleus tractus solitarius. Am J Physiol Regul Integr Comp Physiol 2008, 295:R15-R27.

46. Orina JN, Calcagno AM, Wu CP, Varma S, Shih J, Lin M, Eichler G, Weinstein JN, Pommier Y, Ambudkar SV, Gottesman MM, Gillet JP: Evaluation of current methods used to analyze the expression profiles of ATP-binding cassette transporters yields an improved drug-discovery database. $\mathrm{Mol}$ Cancer Ther 2009, 7:2057-2066.

47. Spurgeon $\mathrm{SL}$, Jones RC, Ramakrishnan R: High throughput gene expression measurement with real time PCR in a microfluidic dynamic array. PLOS One 2008, 2:e1662

48. Fujita A, Sato JR, DeRodrigues LO, Ferreira CE, Sogayar MC: Evaluating different methods of microarray data normalization. BMC Bioinformatics 2006, 7:469.

49. Bolstad BM, Irizarry RA, Astrand M, Speed TP: A comparison of normalization methods for high density oligonucleotide array data based on variance and bias. Bioinformatics 2003, 2:185-193.

50. Pradervand S, Weber J, Thomas J, Bueno M, Wirapati P, Lefort K, Dotto GP, Harshman K: Impact of normalization on miRNA microarray expression profiling. RNA 2009, 3:493-501.

51. Schmittgen TD, Livak KJ: Analyzing real-time PCR data by the comparative C(T) method. Nat Protoc 2008, 6:1101-1108

52. Duijvestijn AM, van Goor H, Klatter F, Majoor GD, van Bussel E, van Breda Vriesman PJ: Antibodies defining rat endothelial cells: RECA-1, a panendothelial cell-specific monoclonal antibody. Lab Invest 1992, 4:459-466.

53. Sims MR: Blood vessel response to pan-endothelium (RECA-1) antibody in normal and tooth loaded rat periodontal ligament. Eur J Orthod 1999, 5:469-479.

54. Saubaméa B, Cochois-Guégan V, Cisternino S, Scherrmann JM: Heterogeneity in the rat brain vasculature revealed by quantitative confocal analysis of endothelial barrier antigen and P-glycoprotein expression. J Cereb Blood Flow Metab 2012, 1:81-92.

55. Macdonald JA, Murugesan N, Pachter JS: Endothelial cell heterogeneity of blood-brain barrier gene expression along the cerebral microvasculature. J Neurosci Res 2010, 7:1457-1474.

56. Seguin R, Biernacki K, Rotondo RL, Prat A, Antel JP: Regulation and functional effects of monocyte migration across human brain-derived endothelial cells. J Neuropathol Exp Neurol 2003, 4:412-419.

57. Turowski P, Adamson P, Greenwood J: Pharmacological targeting of ICAM1 signaling in brain endothelial cells: potential for treating neuroinflammation. Cell Mol Neurobiol 2005, 1:153-170.

58. Wong D, Prameya R, Dorovini-Zis K: Adhesion and migration of polymorphonuclear leukocytes across human brain microvessel endothelial cells are differentially regulated by endothelial cell adhesion molecules and modulate monolayer permeability. J Neuroimmuno/ 2007, 1-2:136-148.

59. Bar KJ, Boettger MK, Schulz S, Neubauer R, Jochum T, Voss A, Yeragani VK: Reduced cardio-respiratory coupling in acute alcohol withdrawal. Drug Alcohol Depend 2008, 3:210-217.

60. Zera T, Ufnal M, Szczepanska-Sadowska E: Central TNF-alpha elevates blood pressure and sensitizes to central pressor action of angiotensin II in the infarcted rats. J Physiol Pharmacol 2008, 59(Suppl 8):S117-S121.

61. Knapp DJ, Whitman BA, Wills TA, Angel RA, Overstreet DH, Criswell HE, Ming Z, Breese GR: Cytokine involvement in stress may depend on corticotrophin releasing factor to sensitize ethanol withdrawal anxiety. Brain Behav Immun 2011, 25(Suppl 1):S146-S154.

62. Pascual M, Balino P, Alfonso-Loeches S, Aragon CM, Guerri C: Impact of TLR4 on behavioral and cognitive dysfunctions associated with alcoholinduced neuroinflammatory damage. Brain Behav Immun 2011, 25(Suppl 1):S80-S91.

63. Koob GF: Theoretical frameworks and mechanistic aspects of alcohol addiction: alcohol addiction as a reward deficit disorder. Curr Top Behav Neurosci 2011. doi:10.1007/7854_2011_129. Epub ahead of print.
64. Collins MA, Corso TD, Neafsey EJ: Neuronal degeneration in rat cerebrocortical and olfactory regions during subchronic "binge" intoxication with ethanol: possible explanation for olfactory deficits in alcoholics. Alcohol Clin Exp Res 1996, 2:284-292.

65. Prendergast MA, Harris BR, Mullholland PJ, Blanchard JA, Gibson DA, Holley RC, Littleton JM: Hippocampal CA1 region neurodegeneration produced by ethanol withdrawal requires activation of intrinsic polysynaptic hippocampal pathways and function of N-methyl-D-aspartate receptors. Neuroscience 2004, 4:869-877.

66. Mechtcheriakov S, Brenneis C, Egger K, Koppelstaetter F, Schocke M, Marksteiner J: A widespread distinct pattern of cerebral atrophy in patients with alcohol addiction revealed by voxel-based morphometry. $J$ Neurol Neurosurg Psychiatry 2007, 6:610-614.

67. Benjamins JA, Nedelkoska L, Lisak RP, Hannigan JH, Sokol RJ: Cytokines reduce toxic effects of ethanol on oligodendroglia. Neurochem Res 2011, 9:1677-1686.

68. Rump TJ, Abdul Muneer PM, Szlachetka AM, Lamb A, Haorei C, Alikunju S, Xiong H, Keblesh J, Liu J, Zimmerman MC, Jones J, Donohue TM Jr, Persidsky Y, Haorah J: Acetyl-L-carnitine protects neuronal function from alcohol-induced oxidative damage in the brain. Free Radic Biol Med 2010, 10:1494-1504

69. Zahr NM, Luong R, Sullivan EV, Pfefferbaum A: Measurement of serum, liver, and brain cytokine induction, thiamine levels, and hepatopathology in rats exposed to a 4-day alcohol binge protocol. Alcohol Clin Exp Res 2010, 34:1858-1870.

70. Zahr NM, Luong R, Sullivan EV, Pfefferbaum A: Coordinated Dynamic Gene Expression Changes in the Central Nucleus of the Amygdala During Alcohol Withdrawal. Alcohol Clin Exp Res doi:10.1111/j.15300277.2012.01910.x

doi:10.1186/1742-2094-9-97

Cite this article as: Freeman et al:: Temporal changes in innate immune signals in a rat model of alcohol withdrawal in emotional and cardiorespiratory homeostatic nuclei. Journal of Neuroinflammation 2012 9:97.

\section{Submit your next manuscript to BioMed Central and take full advantage of:}

- Convenient online submission

- Thorough peer review

- No space constraints or color figure charges

- Immediate publication on acceptance

- Inclusion in PubMed, CAS, Scopus and Google Scholar

- Research which is freely available for redistribution 